\title{
Bone marrow histology 3: Value of bone marrow core biopsy in acute leukaemia, myelodysplastic syndromes, and chronic myeloid leukaemia
}

\author{
D A Winfield, S V Polacarz
}

\section{Introduction}

The standard approach to the diagnosis of acute leukaemia and myelodysplasia has been based on the morphology and percentage of malignant haemopoietic cells in peripheral blood and in an aspirated sample of bone marrow.

The FAB group reports on acute leukaemia have emphasised the value of additional information gained from cytochemistry, ${ }^{1}$ and immunological studies and cytogenetics are increasingly being used to provide an accurate diagnosis in acute leukaemia ${ }^{2}$ and myelodysplasia.

Aspirated bone marrow only provides a small sample which is diluted with sinusoidal blood and provides no information on the architectural changes occurring within the marrow cavity as a result of the leukaemic process. An adequate core biopsy sample permits a more accurate analysis of the degree of involvement of the marrow by the leukaemic process and may provide additional information on the response of normal marrow elements to the malignant infiltrate. Despite these advantages marrow core biopsy is not widely accepted as an essential requirement in the investigation of haemopoietic malignancies. One reason for this may be the view that decalcification and paraffin-wax embedding of such biopsy specimens produces distortion and shrinkage, resulting in difficulty in interpreting cellular morphology. This problem can be overcome by using plastic embedding of biopsy specimens, ${ }^{3}$ but satisfactory morphological details may also be obtained with improved methods of processing paraffin-wax embedded material, ${ }^{4}$, with the additional advantage that such processing also allows the use of immunocytochemical techniques.

This review will assess the value of marrow core biopsy as a routine diagnostic procedure in the investigation of acute leukaemia, myelodysplasia, and chronic myeloid leukaemia.

\footnotetext{
Haematology

Department, Royal

Hallamshire Hospital,

Sheffield S10 2JF

D A Winfield

Department of

Pathology, University

of Sheffield Medical

School

S V Polacarz

Correspondence to:

Dr D A Winfield

Accepted for publication

20 March 1992
} Biopsy material also allows for accurate qu tation of normal marrow components such as plasma cells, lymphocytes, mast cells, eosinophils and macrophages which are sometimes

\section{(AML)} that it provides a more accurate assessment of total marrow cellularity and blast cell numbers both of which are usually numerically greater found in increased numbers in acute leukaemia. ${ }^{6}$ Whether this additional information is of any prognostic value is at present uncertain.

The newer techniques of processing core biopsy specimens make recognition of cytomorphological features easier, but the appearances of cells are often different from those seen in Romanowsky stained marrow smears. Despite these differences it is usually possible on marrow section to distinguish accurately between acute myeloblastic and lymphoblastic leukaemia, but morphological classification into the AML FAB subtypes can be difficult and a comparison of biopsy specimens and aspirates resulted in agreement in only $57 \%$ of cases. $^{7}$

Enzyme histochemistry is routinely used for diagnosis on marrow aspirates but is limited on paraffin-wax embedded sections to the detection of chloroacetate esterase by the Leder procedure. ${ }^{8}$ The use of plastic embedding procedures allows for a wide range of histochemical reactions, including chloroacetate esterase in myeloid cells (fig 1), $a$-naphthyl butyrate esterase in monocyte/macrophage cells, alkaline phosphatase in fibroblast-like reticulum cells and acid phosphatase in megakaryocytes, monocytes, and T lymphocytes. ${ }^{9}$ The usefulness of the information obtained from marrow core enzyme histochemistry requires further evaluation, although the confirmed value of chloroacetate esterase in determining cells of the myeloid series indicates that the Leder procedure should be routinely used on marrow biopsy specimens.

Monoclonal antibodies detecting myeloid antigens are now available for use on paraffin wax sections of marrow biopsy specimens and have been evaluated in the immunophenotyping of AML. ${ }^{1111}$ Positive reactivity is obtained with a range of antibodies but because AML subtypes exhibit a heterogenic phenotype a single antibody rarely produces diagnostically useful information with the exceptions of antiglycophorin $\mathrm{C}$ in erythroleukaemia and both anti-platelet glycoprotein III and anti-factor VIII related antigen in megakaryoblastic leukaemia. Immunophenotyping of marrow sections remains at the research stage, but it is likely that this technique will be of increasing value in routine diagnostic work.

\section{HYPOPLASTIC AML}

In the hypoplastic variant of AML core biopsy of marrow is essential for an accurate diagnosis. The incidence of this condition is about $5 \%$ of all cases of AML. The diagnostic features are a hypocellular marrow (less than 
$50 \%$ cellularity) with more than $30 \%$ primitive myeloid cells (fig 2). It is essential that the cellular morphology is carefully assessed in all hypoplastic core biopsy specimens to avoid mistakes in diagnosis, particularly between hypoplastic AML and hypoplastic anaemia. Antecedent myelodysplastic features are found in one third of cases and there is often a history of previous exposure to leukaemogenic agents. ${ }^{12}{ }^{13}$ The pathogenesis of the hypocellularity is uncertain, but serial biopsy specimens usually have a constant hypocellular pattern, indicating that this condition is a true variant of AML.

Megakaryoblastic leukaemia (FAB M7) and acute myelofibrosis

Are these two terms describing separate entities or are they the same disease? Both are characterised by absence of clinical splenomegaly, pancytopenia, and a rapidly fatal course. Blast cells may be present in the peripheral blood and are characterised as megakaryoblasts by a positive platelet peroxidase reaction at ultrastructural level. An adequate marrow aspirate cannot usually be obtained and diagnosis requires core biopsy with features of increased numbers of blast cells, increased

Figure 1 Bone marrow core biopsy specimen from a case of acute myelomonoblastic leukaemia (FAB M4). Positive staining with naphthol $A S-D$ chloroacetate esterase reaction in myeloblasts (Leder's stain).

Figure 2 Bone marrow core biopsy specimen from a case of hypoplastic $A M L$ showing fatty hypocellular marrow with foci of blast cells (haematoxylin and eosin).

Figure 3 Bone marrow core biopsy specimen from a case of megakaryoblastic leukaemia (FAB M7) with prominent atypical megakaryocytes and blast cells (haematoxylin and eosin).

Figure 4 Pronounced fibrosis in a case of megakaryoblastic leukaemia (FAB M7) (Gomori reticulin stain).

Figure 5 Bone marrow core biopsy specimen from a case of MDS showing ALIP and occasional dyserythropoietic features (1) haematoxylin and eosin).

Figure 6 Bone marrow core biopsy specimen from a case of CML showing paratrabecular blast cells and promyelocytes with maturing myeloid cells in maturing myeloid cells in space (haematoxylin and eosin).

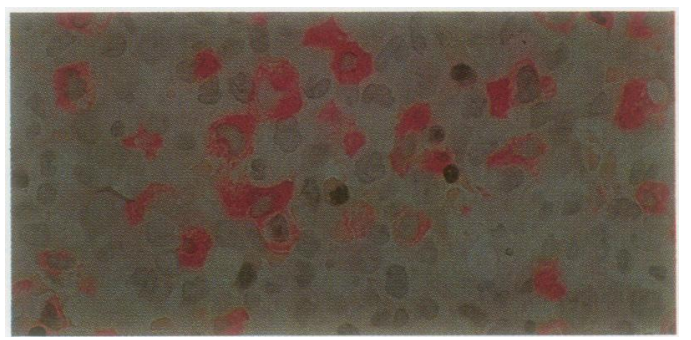

1
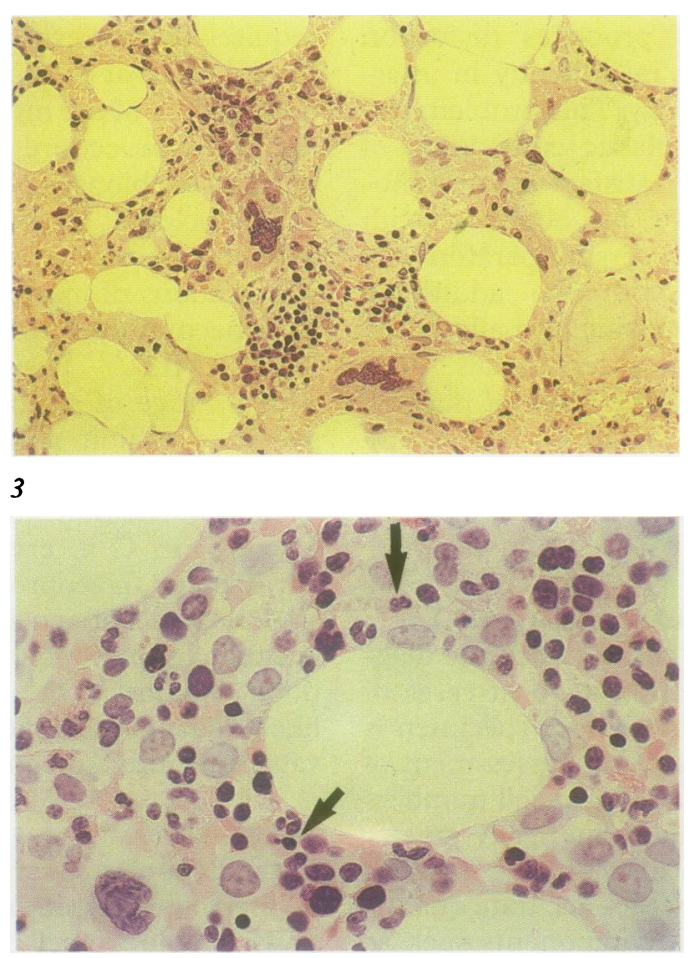

5

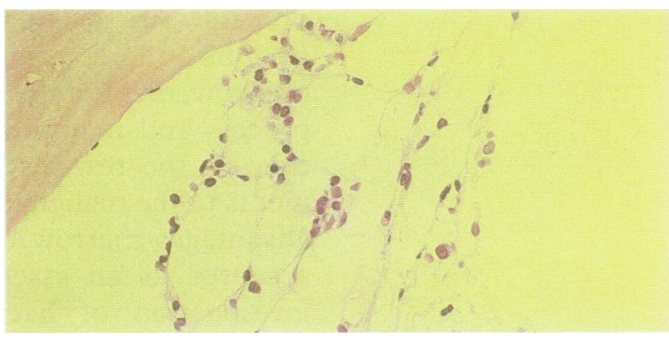

megakaryocytes of atypical morphology, and a dense increase in reticulin (figs 3 and 4).

In a comparative study of FAB M7 and acute myelofibrosis there were similar morphological findings with the exception of more prominent lymphocytic aggregates in acute myelofibrosis. These diseases can be separated into different entities by immunohistochemical reactivity to monoclonal anti-factor VIII related antigen. In FAB $M 7$ the blast cells in the core biopsy specimen are positive; in acute myelofibrosis they are negative. ${ }^{14}$ This finding, together with a previous report of heterogeneity of the blast cells in acute myelofibrosis, ${ }^{15}$ suggests that acute myelofibrosis results from a proliferation of more primitive stem cells.

Increased marrow reticulin fibres may also be associated with cellular dysplastic changes, but this can be considered as a separate entity and will be discussed in the section dealing with myelodysplastic syndromes.

\section{Acute lymphoblastic leukaemia (ALL)}

In most cases of ALL core biopsy provides only limited additional information compared with standard diagnostic techniques used on aspirated marrow or peripheral blood. Marrow

2

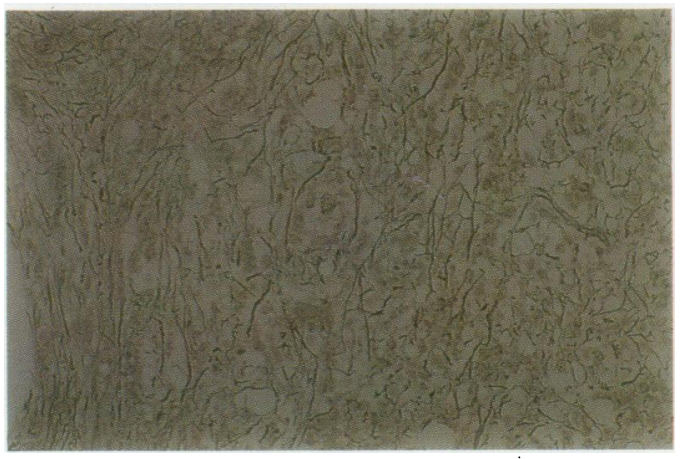

4

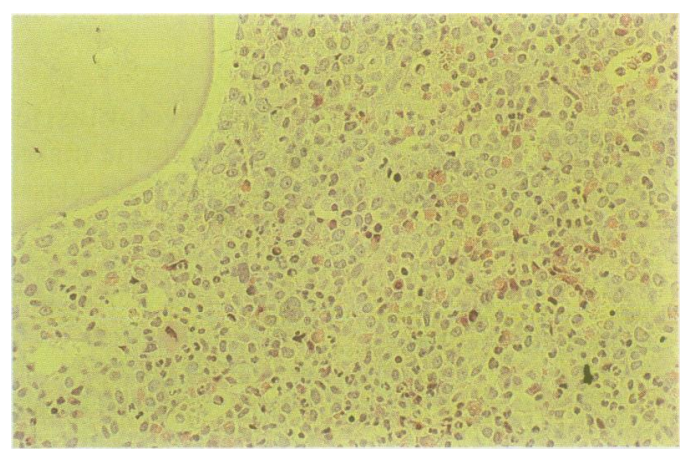


cellularity and blast cell numbers may be more accurately assessed on core biopsy but FAB classification is very difficult. ${ }^{7}$ Increased amounts of reticulin are found in about $70 \%$ of cases of ALL. There is a strong association between an increase in reticulin and lymphoblasts expressing B cell lineage markers, but whether marrow fibrosis is of any prognostic importance is at present uncertain. ${ }^{16}$ There are also a few reported cases of ALL which on core biopsy fulfil the diagnostic criteria for hypoplastic acute leukaemia. ${ }^{13}$ These must be differentiated from the more commonly reported cases of transient marrow aplasia which may precede the development of common ALL. ${ }^{17}$ Bone marrow necrosis can sometimes be suspected in the marrow aspirate of ALL at presentation or relapse because cellular features are lost and background staining is amorphous. In such situations a trephine biopsy specimen is required for confirmation. ${ }^{18}$

Currently, the newer immunohistological techniques are limited in the additional information they can provide and their use is restricted to the detection of T cell ALL with polyclonal anti-CD3. ${ }^{10}$

\section{Myelodysplastic syndromes (MDS)}

The MDS comprise a group of diseases characterised by morphological abnormalities in myeloid, erythroid, and megakaryocytic cell lines. The FAB cooperative group ${ }^{19}$ have defined, by cytological criteria, five subtypes: refractory anaemia (RA), refractory anaemia with ring sideroblasts (RARS); refractory anaemia with excess of blasts (RAEB); refractory anaemia with excess of blasts in transformation (RAEB- $t$ ); and chronic myelomonocytic leukaemia (CMML). Recognition of these subtypes may be difficult in a core biopsy specimen, ${ }^{20}$ although improved accuracy of diagnosis can be achieved if peripheral blood blast cell and monocyte counts are also known. ${ }^{21} \mathrm{~A}$ core biopsy specimen does allow a disturbed marrow cellular spatial orientation to be recognised, resulting in the presence of myeloid precursor cells away from their normal position along endosteal surfaces. This feature is termed abnormal localisation of immature precursors (ALIP) and is defined as the presence of three or more myeloblasts or promyelocytes clustering centrally in the marrow (fig 5). There must be more than three ALIPs per section before they can be considered to be of diagnostic importance, ${ }^{20}$ and it is important to recognise that aggregates of immature erythroid and megakaryocytic cells, as demonstrated by immunochemistry, may also mimic the appearance of an ALIP (pseudoALIP). ${ }^{22}$ Although ALIP is typically associated with MDS, it is not unique to this group of diseases and can sometimes be seen in marrow treated with chemotherapy in AML and chronic myeloid leukaemia, and also following bone marrow transplantation. ${ }^{22}$ Does the finding of ALIP have prognostic as well as diagnostic importance? There seems to be an association between ALIP and shorter patient survival, not only in RAEB, RAEB-t, and CMML where ALIP is most common, but also in RA and RARS where, by definition, there is no excess of myeloblasts in the marrow aspirate. ${ }^{22-24}$

Dysmegakaryopoiesis is found in up to $80 \%$ of cases of $\mathrm{MDS}^{25}$ and abnormal megakaryocytic morphology may be easier to detect in a core biopsy specimen than in aspirated marrow. As well as having diagnostic importance the severity of dysmegakaryopoiesis may also indicate a poorer prognosis. ${ }^{26}$ Characteristic features are hypo- and hyperlobulated megakaryocytes and megakaryoblasts with a high nuclear : cytoplasmic ratio, delicate nuclear chromatin, and one or more nucleoli. ${ }^{20}$ Micromegakaryocytes are frequently present and although they can be recognised in a haematoxylin and eosin preparation, they are more easily seen after staining with periodic acid schiff (PAS) or by immunochemistry using monoclonal antibody anti-CD61 directed against glycoprotein IIIa. ${ }^{25}$ The relatively rare $5 \mathrm{q}$-syndrome is suspected from the finding of a uniform population of monolobulated micromegakaryocytes. ${ }^{20}$

Dyserythropoiesis is present in most cases of $\mathrm{MDS},{ }^{20}$ normally manifest as megaloblastic maturation or as defective maturation in which the erythron is composed of cells at the same stage of development. A predominance of proerythroblasts can also be seen. ${ }^{2026}$

Accurate quantitation of marrow blast cells is required for a correct subtyping of MDS and for differentiation from acute leukaemia. Although quantitation is more difficult in a core biopsy specimen than in aspirated marrow, a good concordance in blast cell estimation between core and aspirate samples has been reported. ${ }^{21}$

A core biopsy specimen is of special value in the detection of increased concentrations of marrow reticulin, present in about $50 \%$ of samples of MDS. ${ }^{202126}$ This is usually of moderate degree, although several recent reports have described cases of myelodysplastic syndrome associated with a pronounced degree of fibrosis. ${ }^{27-29}$ The prognostic importance of this finding is at present unclear because it is suggested that this condition may be associated with an increased ${ }^{27}$ or a decreased $^{2829}$ survival. A biopsy specimen is also important for accurate diagnosis of the rare MDS hypoplastic variant, characterised by pancytopenia, marrow hypoplasia, and a low incidence of progression to acute leukaemia. $^{30}$

The assessment of a biopsy sample in MDS requires not only consideration of the dyshaemopoietic features but should also include quantitation of eosinophils and plasma cells. Eosinophilia, defined as greater than eight eosinophils per microscopic field ( $\times 400$ magnification), has been recorded in a quarter of cases and may be associated with improved survival. Plasmacytosis of more than two plasma cells per microscopic field ( $\times 400$ magnification) is seen in $18 \%$ of all MDS 
cases, and in as many as one half of the CMML subtype, although no prognostic value has been attached to this finding. ${ }^{2126}$

\section{Chronic myeloid leukaemia (CML)}

The diagnosis of CML is based on typical morphological features of excess myeloid activity in blood and bone marrow aspirate, a low leucocyte alkaline phosphatase score, the presence of the Philadelphia chromosome [t $(9 ; 22(\mathrm{q} 34 ; \mathrm{q} 11)]$ and the molecular consequences of the $\mathrm{Ph}$ translocation with formation of the BCR-ABL fusion gene on chromosome 22. A core biopsy specimen provides only limited additional diagnostic information although it has been suggested that two subtypes can be identified. One has proliferation of predominantly granulocytic precursor cells, the other a mixed proliferation of granulocytic and megakaryocytic lines (chronic megakaryocytic-granulocytic myelosis). ${ }^{31}$ The suggestion was made that these morphological subtypes show differences in clinical behaviour, but more recent work has shown no clinical, evolutionary,or prognostic differences between the two groups. ${ }^{32}$

The characteristic morphological pattern in chronic myeloid leukaemia is of blast cells and promyelocytes located in the paratrabecular and perivascular region with increasing proportions of more mature myeloid cells in the central intertrabecular spaces (fig 6). Accurate determination of blast cells and promyelocytic numbers may provide independent prognostic information. Patients with pronounced infiltration (four to eight layers of blasts and promyelocytes) compared with minimal infiltration (one to three layers) have an increased probability of early blast cell transformation. ${ }^{33}$

An advantage of marrow biopsy in CML is that it allows marrow fibrosis to be assessed. In newly diagnosed patients up to half have increased amounts of reticulin in more than $50 \%$ of the cellular areas of the biopsy specimen. Increased fibrosis is strongly associated with poor prognostic features such as splenomegaly, anaemia, increased peripheral blood and marrow blasts, and additional karyotypic abnormalities, and it has therefore been suggested that fibrosis can be a useful prognostic factor at diagnosis. ${ }^{34}$ In sequential biopsy specimens taken during the course of the disease many patients show a progressive increase in marrow fibrosis which correlates with the number of megakaryocytes, and this too is associated with a worsening prognosis. ${ }^{35}$

\section{Conclusion}

A core biopsy specimen complements the peripheral blood and marrow aspirate findings in providing additional information for the diagnosis and assessment of prognosis in acute leukaemias, myelodysplastic syndromes, and chronic myeloid leukaemia. In recommending the routine use of core biopsy it is essential that adequate, well processed samples are available for morphological interpretation and for the assessment of newer cytochemical and immunohistological techniques. Also important is a greater awareness of the benefits of close cooperation between histopathologists and haematologists in ensuring that maximal information is gained from bone marrow samples in haematological malignancies.

SV Polacarz is supported by the Yorkshire Cancer Research Campaign. We are grateful to Mrs J Peel and Mrs M Raymond for their help in preparing the manuscripts.

1 Bennett JM, Catovsky D, Daniel MT, et al. Proposed revised criteria for the classification of acute myeloid
leukemia. A report of the French-American-British leukemia. A report of the French-American-British
Cooperative Group. Ann Intern Med 1985;103:626-9.

2 Second MIC Cooperative Study Group. Morphologic, immunologic, and cytogenetic (MIC) working classification of the acute myeloid leukemias. Cancer Genet Cytogenet 1988;30:1-15.

3 Islam A, Frisch B. Plastic embedding in routine histology I: Preparation of semi-thin sections of undecalcified marrow cores. Histopathology 1985;9:1263-74.

4 Gatter KC, Heryet A, Brown DC, Mason DY. Is it necessary to embed bone-marrow biopsies in plastic for haematological diagnosis? Histopathology 1987;11:1-7.

5 Vincic L, Weston S, Riddell RH. Bone core biopsies. Plastic or paraffin? Am $\mathcal{F}$ Surg Pathol 1989;13:329-34.

6 Islam A, Frisch B, Henderson ES. Plastic embedded core biopsy: a complementary approach to bone marrow aspiration for diagnosing acute myeloid leukaemia. $\mathcal{f}$ Clin Pathol 1989;42:300-6.

7 Dominis M, Džebro S. Histology of bone marrow in acute leukaemia. Bone Marrow Transplant 1989;4 (Suppl 3): $10-11$

8 Leder LD. The chloroacetate esterase reaction. A useful means of histological diagnosis of hematological disorders from paraffin sections of skin. Am $\mathcal{f}$ Dermatopathol from paraffin

9 Beckstead JH, Halverson PS, Ries CA, Bainton DF. Enzyme histochemistry and immunohistochemistry on biopsy specimens of pathologic human bone marrow. Blood 1981;57:1088--96.

10 Kurec AS, Cruz VE, Barrett D, Mason DY, Davey FR. Immunophenotyping of acute leukemias using paraffinembedded tissue sections. Am $\mathcal{f}$ Clin Pathol 1990; 93:502-9.

11 Horny H-P, Campbell M, Steinke B, Kaiserling E. Acute myeloid leukemia: Immunohistologic findings in paraffinembedded bone marrow biopsy specimens. Hum Pathol 1990;21:648-55.

12 Needleman SW, Burns CP, Dick FR, Armitage JO. Hypoplastic acute leukemia. Cancer $1981 ; 48: 1410-14$

13 Berdeaux DH, Glasser L, Serokmann R, Moon T, Durie BGM. Hypoplastic acute leukemia: Review of 70 cases with multivariate regression analysis. Hematol Oncol with multivariate

14 Hruban RH, Kuhajda FP, Mann RB. Acute myelofibrosis. Immunohistochemical study of four cases and comparison with acute megakaryocytic leukemia. $\mathrm{Am} \mathcal{f}$ Clin Pathol 1987;88:578-88.

15 Sultan C, Sigaux F, Imbert M, Reyes F. Acute myelodysplasia with myelofibrosis: a report of eight cases. $\mathrm{Br} \mathscr{Y}$ Haematol 1981;49:11-16.

16 Wallis JP, Reid MM. Bone marrow fibrosis in childhood acute lymphoblastic leukaemia. F Clin Pathol 1989; 42:1253-4.

17 Breatnach F, Chessells JM, Greaves MF. The aplastic presentation of childhood leukaemia: a feature of compresentation of childhood leukaemia: a featur

18 Habboush HW, Hann IM. Bone marrow necrosis in acute lymphoblastic leukaemia. Scot Med f 1987;32:177-80.

19 Bennett JM, Catovsky D, Daniel MT, et al. The FrenchAmerican-British (FAB) Cooperative Group. Proposals for the classification of myelodysplastic syndromes. $\mathrm{Br} \mathcal{F}$ Haematol 1982;51:189-99.

20 Tricot G, De Wolf-Peeters C, Hendrickx B, Verwilghen RL. Bone marrow histology in myelodysplastic syndromes 1 . Histological findings in myelodysplastic syndromes and comparison with bone marrow smears. Br $f$ Haematol 1984;57:423-30.

21 Delacrétaz F, Schmidt P-M, Piguet D, Bachmann F, Costa $\mathrm{J}$. Histopathology of myelodysplastic syndromes. The FAB classification (proposals) applied to bone marrow biopsy. Am $¥$ Clin Pathol 1987;87:180-6.

22 Mangi MH, Salisbury JR, Mufti GJ. Abnormal localisation of immature precursors (ALIP) in the bone marrow of of immature precursors (ALIP) in the bone marrow of
myelodysplastic syndromes: current state of knowledge myelodysplastic syndromes: current state of know

23 Mangi MH, Mufti GJ. Primary myelodysplastic syndromes: diagnostic and prognostic significance of immunohistochemical assessment of bone marrow biopsies. Blood 1992;79:198-205

24 Tricot G, De Wolf-Peeters C, Vlietinck R, Verwilghen RL. Bone marrow histology in myelodysplastic syndromes II. Prognostic value of abnormal localization of immatur precursors in MDS. Br f Haematol 1984;58:217-25. 25 Thiele J, Quitmann H, Wagner S, Fischer R. Dysmega- 
karyopoiesis in myelodysplastic syndromes (MDS): an immunomorphometric study of bone marrow trephine biopsy specimens. $\mathcal{F}$ Clin Pathol 1991;44:300-5.

26 Rios A, Cañizo MC, Sanz MA, et al. Bone marrow biopsy in myelodysplastic syndromes: morphological characteristics and contribution to the study of prognostic factors. $\mathrm{Br}$ f Haematol 1990;75:26-33.

27 Pagliuca A, Layton DM, Manoharan A, Gordon S, Green PJ, Mufti GJ. Myelofibrosis in primary myelodysplastic PJ, Mufti GJ. Myelofibrosis in primary myelodysplastic
syndromes: a clinicomorphological study of 10 cases. $\mathrm{Br} F$ Syndromes: a clinicomorphol

28 Lambertenghi-Deliliers G, Orazi A, Luksch R, Annaloro C, Soligo $\mathrm{D}$. Myelodysplastic syndrome with increased marrow fibrosis: a distinct clinico-pathological entity. $\mathrm{Br} \mathcal{F}$ Haematol 1991;78:161-6.

29 Takahashi M, Koike T, Nagayama R, et al. Myelodysplastic syndrome with myelofibrosis: myelodysplastic syndrome as a major primary disorder for acute myelofibrosis. Clin Lab Haematol 1991;13:17-23.

30 Nand S, Godwin JE. Hypoplastic myelodysplastic syn- drome. Cancer 1988;62:958-64.

31 Georgii A, Vykoupil KF, Thiele J. Classification of chronic myeloproliferative diseases by bone marrow biopsies. Hematological and cytogenetic findings and clinical course. Bibl Haematol 1984;50:41-56.

32 Rozman C, Cervantes F, Feliu E. Is the histological classification of chronic granulocytic leukaemia justified from the clinical point of view? Eur $\mathcal{f}$ Haematol 1989; 42:150-4.

33 Islam A. Prediction of impending blast cell transformation in chronic granulocytic leukaemia. Histopathology 1988; 12:633-9.

34 Dekmezian R, Kantariian HM, Keating MJ, Talpaz M, McCredie KB, Freireich EJ. The relevance of reticulin stain-measured fibrosis at diagnosis in chronic myelogenous leukemia. Cancer 1987;59:1739-43.

35 Lazzarino M, Morra E, Castello A, et al. Myelofibrosis in chronic granulocytic leukaemia: clinicopathologic correlations and prognostic significance. $\mathrm{Br} \mathcal{f}$ Haematol 1986; 64:227-40. 